\title{
Estimación de Normales y Reducción de Datos Atípicos en Nubes de Puntos Tridimensionales
}

\author{
Esmeide Leal $^{(1) \star}$, Nallig Leal ${ }^{(2)}$ y German Sánchez ${ }^{(3)}$ \\ (1) Universidad Autónoma del Caribe, Programa Análisis y Diseño de Sistemas y Computación. Calle 90 \\ No. 46-112,Barranquilla - Colombia (e-mail: esleal@uac.edu.co). \\ (2) Universidad Autónoma del Caribe, Programa Ingeniería de Sistemas. Calle 90 No. 46-112, Barranquilla \\ - Colombia (e-mail: nleal@uac.edu.co). \\ (3) Universidad del Magdalena, Programa de Ingeniería de Sistemas. Carrera 32 No. 22-08, Santa Marta - \\ Colombia (e-mail: gsanchez@unimagdalena.edu.co).
}

${ }^{*}$ Autor a quien debe ser dirigida la correspondencia.

Recibido Sep. 10, 2013; Aceptado Nov. 28, 2013; Versión final recibida Dic. 3, 2013

\begin{abstract}
Resumen
En este trabajo se presenta un método para la estimación de los vectores normales y para la reducción de datos atípicos en las superficies descritas a partir de puntos 3D. El método se basa en la técnica de Análisis de Componentes Principales Robusto que es aplicada a un vecindario estimado de acuerdo a las condiciones geométricas locales. Inicialmente se eliminan los datos atípicos con un procedimiento basado en la distancia de Mahalanobis. Posteriormente, para cada uno de los puntos restantes el método toma un vecindario de tamaño variable. Se muestra que la estimación del plano tangente es más precisa utilizando un vecindario de tamaño variable en relación con uno fijo, permitiendo una estimación más precisa de la normal. El método permite estimar los vectores normales de manera robusta al ruido, datos atípicos y presencia de bordes y esquina.
\end{abstract}

Palabras clave: estimación de normales, nube de puntos, reconstrucción tridimensional.

\section{Surface Normal Estimation and Outliers Reduction on Tridimensional Cloud Points}

\begin{abstract}
This paper shows a method for normal vectors estimation and outlier reduction for point cloud surfaces. This method is based on Robust Principal Component Analysis applying to a sizable neighborhood, estimated according to local geometric featuring. First, outlier reduction is applied by means of a Mahalanobis distance based procedure. Subsequently, a variable size neighborhood is estimated calculating local geometric features. The resizable neighborhood showed to give a more accurate tangent plane estimation than a size fixed neighborhood, allowing accurate normal estimation. The method permits normal vector estimation that is robust to noise, outliers and border and sharp features.
\end{abstract}

Keywords: normal vector estimation, cloud points, surface reconstruction 


\section{INTRODUCCIÓN}

Los modernos dispositivos de adquisición de información tridimensional se han convertido en herramientas de gran precisión y utilidad, por lo que cada vez son más populares para diversas tareas en diferentes campos de aplicación. Estos dispositivos, están diseñados para realizar un detallado escaneo de objetos complejos, generando una gran cantidad de puntos comúnmente llamados Nube de Puntos no Organizados (Cuesta et al. 2008; Risent et al. 2013). Esta nube de puntos es una representación digital y discreta de los objetos escaneados en la memoria del computador. Una vez en la memoria del computador, y como punto de partida para múltiples aplicaciones, es necesario el diseño e implementación de algoritmos para la reconstrucción y segmentación de superficies, detección de bordes y generación de imágenes, entre otros. El buen desempeño de estos algoritmos depende de la correcta estimación de los vectores normales.

Un paso común en los algoritmos de estimación de normales es determinar un vecindario alrededor de cada punto para aproximar la superficie localmente. Son muchos los algoritmos usados en reconstrucción de superficies que toman un vecindario de tamaño fijo, el criterio para la elección del tamaño por lo general lo determinan de manera experimental ignorando la variación de la curvatura y la densidad de muestreo (Mederos et al., 2003; Alexa et al., 2001; Hoppe et al., 1992).En el trabajo de Hoppe et al. (1992), a cada punto se le estima un plano tangente mediante la técnica de Mínimos Cuadrados usando un vecindario formado por los $k$ vecinos más cercanos, donde $k$ es seleccionado experimentalmente. Este método falla cuando la nube de puntos contiene ruido y valores atípicos, ya que el plano de regresión se estima usando Análisis de Componentes Principales (PCA), como en Pauly et al. (2002), Fleishman et al. (2005) y Bao et al. (2010).En el trabajo de Mitra et al. (2004), se realiza un análisis de valores propios y se agrupan los puntos utilizando un algoritmo de Aproximación de vecindarios, para luego analizar la densidad de muestreo y la curvatura, logrando así que éste llegue a ser adaptativo. Sin embargo, su aproximación depende de parámetros ingresados por los usuarios y la aproximación mediante la técnica PCA es sensible al ruido ni a los valores atípicos.

Un enfoque común es estimar una aproximación de la superficie mediante funciones matemáticas (Fleishman et al., 2005; Boulch y renaud, 2012; Prabhakar et al. 2013), o mediante la estimación de una malla triangular. Los primeros, utilizan el concepto de Mínimos Cuadrados Móviles (MLS) (Levin, 1998), lo cual las hace robustas al ruido y a los valores atípicos. Sin embargo, los métodos basados en MLS tienen el inconveniente de incorporar un excesivo suavizado de la superficie. En los métodos basados en mallas triangulares, la estimación de la normal en un vértice $v_{i}$, se obtiene a partir del promedio de las normales a cada una de las caras o triángulos adyacentes a $v_{i}$ (Woo et al., 2002).Con frecuencia, la triangulación inicial es estimada mediante los métodos de Delaunay/Voronoi (Alliez et al., 2007). Dey y Sun, (2006), muestran como las normales pueden ser aproximadas a partir de los diagramas de Voronoi. Específicamente, se muestra que los vértices más alejados, a partir de los sitios los cuales llaman polos, proveen una buena estimación de la normal, sin embargo este método supone que no existe ruido en los datos. El concepto de polo es extendido a datos ruidosos por Dey y Goswami (2006). Ouyan y Feng (2005), construyen un enmallado local basado en diagramas 3D de Voronoiy proponen una regla heurística de crecimiento de mallas para ajustar un grupo de curvas cuadráticas por medio de la cuales se obtienen los vectores tangentes. Sin embargo, la mayor limitación de estos métodos es que no describen un manejo de la normal en los bordes ni en esquinas.

En general, las propuestas anteriores plantean soluciones adecuadas al problema de la estimación aproximada de la normal a la superficie por medio de mallas triangulares o regresiones cuadráticas. Sin embargo, es deseable soluciones que además de ser tolerantes al ruido, a los valores atípicos, operen exclusivamente sobre los puntos, para evitar el costo computacional del cálculo de la triangulación. Este trabajo presenta un método para la estimación de normales en superficies basadas en puntos. El método propuesto toma en cuenta las características implícitas de la superficie como el ruido en la nube de puntos y la variación de la curvatura para estimar el tamaño del vecindario. El método propuesto también analiza la estimación de la normal en partes con picos y filos, como esquinas y bordes.

\section{MATERIALES Y MÉTODOS}

Para estimar las normales se procede en dos etapas: la eliminación de datos atípicos y la estimación del tamaño del vecindario.

\section{Detección y eliminación de valores atípicos}

Los valores atípicos pueden afectar significativamente la precisión de la estimación del vector Normal, por lo tanto es deseable un procedimiento para detectarlos y eliminarlos del conjunto de datos. Para la detección de dichos valores atípicos se utilizó la Distancia Mahalanobis (DM). La DM tiene en cuenta la varianza de 
los datos y por lo tanto mide la dispersión de los datos respecto a la media. Para una muestra de datos multivariada $n$-dimensional, la Distancia Mahalanobis se define como:

$D M\left(p_{i}\right)=\left(p_{i}-\bar{p}_{w}\right)^{T} C M_{w}^{-1}\left(p_{i}-\bar{p}_{w}\right)$

Donde $C M_{w}^{-1}$ es la matriz pesada de la covarianza inversa del vecindario del punto $p_{i}$ y $\bar{p}_{w}$ es la media pesada.Para datos multivariados distribuidos normalmente, los valores de $D M^{2}$ se distribuyen aproximadamente como una ji-Cuadrada con $n$-grados de libertad $\left(\chi_{n}^{2}\right)$. Esto es, configurando $D M^{2}$ a una constante dada con un cierto cuantil de $\chi_{n}^{2}$. Se pueden considerar como valores atípicos, aquellos cuya DM a la media del vecindario está por fuera en un intervalo de confianza $\chi_{n ; 1-\alpha}^{2}$. Es decir, $D M\left(p_{i}\right)>\chi_{p ; 1-\alpha}^{2}$, lo queconstituye un método de detección de valores atípicos multivariado basado en el método propuesto en Filzmosera et al. (2005), y mejorado incluyendo la matriz de covarianzas estimada de forma robusta $C M_{w}$ descrita en la ecuación (1), mejorando así $D M\left(p_{i}\right)$.

\section{Estimación de vectores normales}

El problema de la estimación de los vectores normales se divide en dos etapas: La determinación del tamaño del vecindario de cada uno de los puntos y la corrección de la estimación en bordes y esquinas. Para determinar el tamaño adecuado se inicia tomando un vecindario grande de puntos que se disminuye iterativamente hasta que se cumpla un criterio prestablecido. Una vez definido, se estima un plano tangente al vecindario y finalmente la Normal.

El problema puede definirse como: dado un conjunto de puntos $P=\left\{(x, y, z)^{T}\right\} \subset R^{3}$, los cuales probablemente fueron muestreados con ruido a partir de una curva o superficie desconocida $S$, esto es $z_{i}=f\left(x_{i}, y_{i}\right)+\varepsilon_{i}$, donde $\varepsilon_{i}$ es el ruido en las muestras. El objetivo es estimar la normal en cada punto $P_{i}=\left(x_{i}, y_{i}, z_{i}\right)$ minimizando el error en relación con la normal verdadera.

Como punto de partida optamos por obtener los $k$-vecinos más cercanos a cada $P_{i}$. Una vez obtenido el vecindario, el siguiente paso es ajustar un plano a la superficie utilizando Análisis de Componentes Principales Robusto, como se muestra a continuación:

$C M_{w}=\frac{1}{n-1} \sum_{i=1}^{n}\left(p_{i}-\bar{p}_{w}\right)\left(p_{i}-\bar{p}_{w}\right)^{T} W$

$\bar{p}_{w}=\frac{\sum_{i=1}^{n} w_{i} p_{i}}{\sum_{i=1}^{n} w_{i}}$

$w_{i}=\frac{1}{d_{i} \cdot \sum_{j=1}^{n} \frac{1}{d_{j}}}$

donde, $C M_{w}$ es la matriz pesada de covarianzas, los $w=\left\{\sqrt{w_{1}}, \cdots, \sqrt{w_{n}}\right\}$, son los pesos asociados a cada punto del vecindario de $p_{i}$ y $\bar{p}_{w}$ es la media pesada. Los pesos $w_{i}$ son calculados utilizando una repartición inversa, donde $d_{i}$ es la distancia Euclidea entre la media del vecindario y cada punto $p_{i}$.De esta manera, se tiene una versión robusta del PCA, denominada RPCA (Hubert et al., 2005), esta versión es capaz de manejar el ruido que esté presente en la nube de puntos. Un factor de influencia en la estimación de las normales es la elección de un tamaño adecuado del vecindario. La figura 1, ilustra la influencia en la estimación del vector normal por la escogencia de un tamaño adecuado e inadecuado del vecindario.

Cuando se escoge un vecindario muy grande la superficie estimada resulta excesivamente suavizada produciendo la pérdida de detalles finos y dificultando la distinción de la separación de dos superficies adyacentes. Por el contrario, si se toma un vecindario pequeño se produce una estimación imprecisa de la Normal, principalmente en la vecindad de regiones con altas variaciones geométricas (esquinas, bordes, entre otros). Esta imprecisión es aún mayor cuando los datos muestreados poseen ruido.

\section{Vecindario Adaptativo}

Para una mejor comprensión del método y sin pérdida de generalización, usaremos como ejemplo una variedad en $R^{2}$ en lugar de una en $R^{3}$. En la estimación adecuada de la normal, también intervienen la curvatura en un punto dado, para encontrar la relación entre el tamaño del vecindario alrededor de un punto 
$p_{i}$, y la curvatura en éstese hará la siguiente relación apoyada en la definición encontrada en Aleksandrov et al. 1984. La curvatura en un punto está dada pork $=\lim _{l \rightarrow 0} \frac{2 h}{l^{2}}$, donde $h$ es la distancia de un segundo punto $p_{i}$ de la curva a la tangente en el punto dado $o$, I es la longitud del segmento de tangente comprendido entre el punto de tangencia oy la proyección del punto $p_{i}$ sobre ella (ver figura 2a).
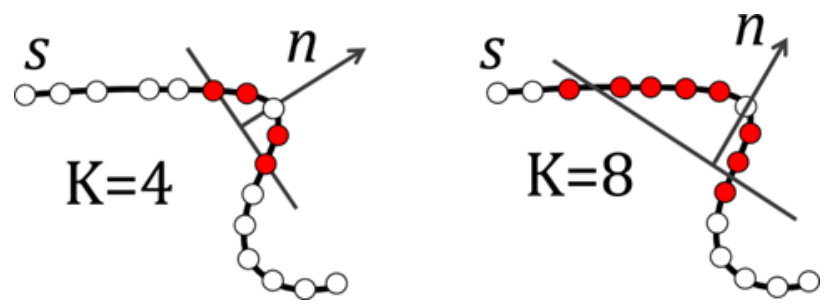

Fig. 1. Influencia del tamaño del vecindario sobre la estimación del vector normal.

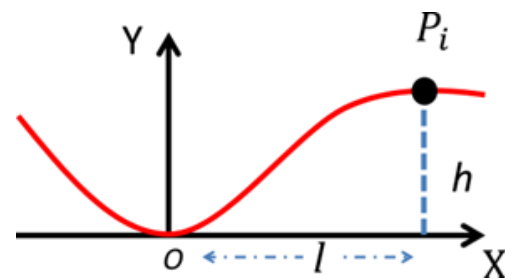

a)

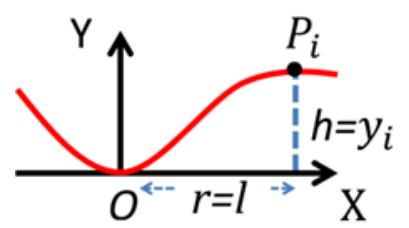

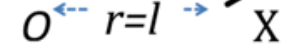

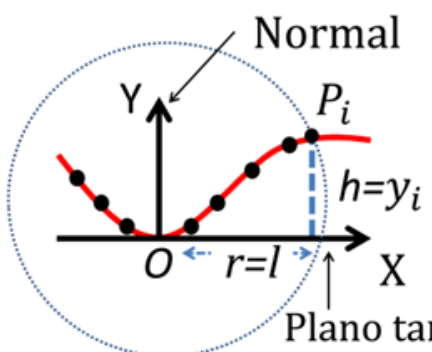

b)

Fig. 2.a) Aproximación de la curvatura en $o$, b) Relación entre el vecindario y la curvatura en un punto $p_{i}$

Para lo anterior, se elige un sistema de coordenadas rectangulares tal que el origen sea el punto $p_{i}$ de la curva, y el eje $\overrightarrow{O X}$ la tangente en ese punto, entonces $y^{\prime}=0$ y $k=\left|y^{\prime \prime}\right|$, desarrollando la función $y=$ $f(x)$ por la fórmula de Taylor, obtenemos $y=\frac{1}{2} y^{\prime \prime} x^{2}+\varepsilon x^{2}$, ya que $y^{\prime}=0$. Tenemos que $\varepsilon \rightarrow 0$ cuando $x \rightarrow 0$, de aquí se tiene que $k=\left|y^{\prime \prime}\right|=\lim _{x \rightarrow 0} 2|y| / x^{2}$, por lo tanto, como $|y|=h, x^{2}=l^{2}$, se tiene $k=\lim _{l \rightarrow 0} \frac{2 h}{l^{2}}$, esta fórmula de curvatura muestra o describe la rapidez con que la curva abandona la tangente.Partiendo de lo anterior se puede llevar este modelo a nuestro problema del vecindario como lo muestra la figura $2 \mathrm{~b}$.

La idea intuitiva de la relación entre las proyecciones al plano de cada punto perteneciente al vecindario y el tamaño adecuado para éste consiste en que si la curvatura es muy pronunciada el tamaño del vecindario tiende a ser pequeño, lo contrario es, si la curvatura es tenue el tamaño del vecindario tiende a ser más grande. Así, para una curvatura pronunciada, el radio del vecindario $r_{i}$ es mucho menor que la proyección de un punto dado $y_{i}$, es decir $\left(r_{i}<y_{i}\right)$, lo contrario ocurre cuando la curvatura es pequeña; la proyección $y_{i}$ es más pequeña en comparación con el radio $\left(r_{i}>y_{i}\right)$. Una vez obtenidos los vectores propios a partir de la matriz de covarianza, el próximo paso es la proyección de los vectores formados por cada uno de los $p_{i}$ y el centroide $\bar{p}_{i}$ de cada vecindario $\left(p_{i}-\bar{p}_{i}\right)$. La obtención de dicha proyección es dada por la expresión conocida del álgebra lineal $P_{v} u=\langle u \mid v\rangle \vec{v}$, lo que dice que es la proyección del vector $u$ sobre el $v$, en dirección de $v$, la cual se denota como $P_{\text {roy }}\left(p_{i}-\bar{p}\right)$.

Se busca una expresión que relacione la curvatura en el punto origen $o$, con las alturas $n\left(p_{i}-(\bar{p}+t n)\right)$ de los $p_{i}$ y el radio del vecindario $r_{i}=\max \left\|P_{\text {roy }}\left(p_{i}-\bar{p}\right)\right\|$. Siendon el vector normal y $t$ un desplazamiento del punto $\bar{p}$ en dirección de la normal, el cual evita el sesgo en los datos estimados. Para esto, se considera que el segmento de curva cerca de $o$ (ver figura 2b) es la gráfica de una función de tipo $C^{2}$ con $y=f(x)$, definida sobre un intervalo $R$ dado por[ $[-r, r]$, y $\left|f^{\prime \prime}(x)\right|<k$ para todos $\operatorname{los} x \in R$, donde $k$ es una constante positiva, en nuestro caso la curvatura en el origen $o$. Es así que para todox $\in[-r, r]$ existe una $k>0$, para la cual $\left|f^{\prime \prime}(x)\right|<k$ ó $\left|y^{\prime \prime}\right|<k$. Lo anterior constituye una cota superior para la curvatura en el intervalo [-r, $r$, para todos los valores de la curvatura de los $p_{i}$ que constituyen el vecindario.

Partiendo de $k=\frac{2 h}{l^{2}}=\frac{2 y}{r^{2}}$, se debe asumir vecindarios donde la curvatura sea pequeña; es decir, $r_{i} \gg y_{i}$,es posible establecer que $\left|y_{i}\right| \leq k \frac{r_{i}^{2}}{2}$, ya que el término $k \frac{r_{i}^{2}}{2}$ identifica una parábola en el origen $o$, en la cual la ordenada $y_{i}$ es menor que la abscisa $r_{i}=x_{i} ;\left(y_{i}<r_{i}\right)$, en el intervalor $r_{i} \in[-r, r]$, y por consiguiente $\left|y_{i}\right| \leq$ 
$k \frac{r_{i}^{2}}{2}$, lo cual establece un criterio de adaptabilidad, que produce que el vecindario varíe dependiendo de la curvatura en el punto establecido. De lo anterior se tiene que:

$\left\|n\left(p_{i}-(\bar{p}+t n)\right)\right\| \leq \frac{k}{2}\|\operatorname{Proy}(P-\bar{P})\|^{2}$

La curvatura $k$ (máxima curvatura), en el punto $p_{i}$, se aproxima utilizando (Pauly et al., 2002):

$C=\frac{\lambda_{0}}{\lambda_{0}+\lambda_{1}}, \operatorname{con} \lambda_{0} \leq \lambda_{1}$

y $\lambda_{0}, \lambda_{1}$ son los valores propios de la matriz $C M_{w}$.

La expresión 5 opera de la siguiente manera: se toma un vecindario lo suficientemente grande, si la desigualdad no se cumple se quita una cantidad predefinida de puntos del vecindario hasta que se cumpla. El resultado de esta iteración, es el tamaño adecuado del vecindario para el punto $p_{i}$ en cuestión.

\section{Corrección de Normales en Bordes y Esquinas}

Un problema subyacente en el algoritmo del PCA con el cual se aproxima el plano tangente del vecindario, es el concerniente a la imprecisa estimación de la normal en partes de la superficie donde existen discontinuidades tales como: bordes, aristas y esquinas. Esto es causado por la incorporación de puntos que pertenecen a ambos lados de la discontinuidad. Al utilizar puntos a ambos lados, lo que se hace es un promedio de las normales, provocando que no se aproxime la normal correctamente. Una solución a éste problema es buscar un vecindario consistente en el que sólo estén puntos a un único lado de la discontinuidad, de manera que permita estimar correctamente la normal en estas partes. Para encontrar un vecindario consistente, primero se deben identificar los puntos que caen en dichas discontinuidades a los cuales se le llamará ambiguos, ya que no se sabe con certeza a qué lado de la discontinuidad pertenecen.

Los puntos no ambiguos, son aquellos que caen al interior de una superficie relativamente plana, ésta puede ser identificada utilizando RPCA en una representación en $\mathrm{R}^{3}$, el cual produce tres valores propios $\lambda_{0} \leq \lambda_{1} \leq \lambda_{2}$ y tres vectores propios $v_{0}, v_{1}, v_{2}$ (los cuales se pueden ver como un elipsoide de correlación).Para un conjunto de puntos que caen en una superficie relativamente plana, el primer vector propio es la aproximación de la normal y su correspondiente valor propio $\lambda_{0}$ será aproximadamente cero. Lo contrario ocurre cuando un conjunto de puntos se encuentra sobre una discontinuidad, puede observarse que $\lambda_{0}$ será grande en comparación con $\lambda_{2}$. Es por eso que el cociente entre $\lambda_{0}$ y $\lambda_{2}$, puede ser usado como una medida de probabilidad de que el punto se encuentre cerca de una discontinuidad en la superficie. $\mathrm{O}$ sea si $\frac{\lambda_{0}}{\lambda_{2}}>\varepsilon$ entonces se trata de un punto ambiguo, esta prueba hay que realizarla con todos los puntos pertenecientes a la superficie para su clasificación. Una vez establecidos los puntos ambiguos, el paso a seguir es identificar el tipo de discontinuidad (esquina, borde, o arista). En Gumhold et al. (2001), un punto se encuentra en una arista, si el elipsoide de correlación se estrecha en dirección de la arista, los valores propios cumplen $\lambda_{0} \approx \lambda_{1}$ y $\lambda_{0}+\lambda_{1} \approx \lambda_{2}$.Si es un punto perteneciente a un borde, el elipsoide degenera en una elipse. El vector propio más pequeño es aproximadamente cero, los otros dos valores propios cumplen $2 \lambda_{1} \approx \lambda_{2}$, el vector propio $v_{2}$ da la dirección del borde.En una esquina, el elipsoide de correlación no tiene una dirección específica los valores propios cumplen $\lambda_{0} \approx \lambda_{1} \approx \lambda_{2}$. Una vez obtenidos los tipos de discontinuidades, se procede a realizar una agrupación por $k$-medias de los puntos alrededor de ésta. Si la discontinuidad es un borde o una arista serán dos agrupaciones, por el contrario si la discontinuidad es una esquina serán tres agrupaciones. El próximo paso es encontrar el grupo más cercano al punto ambiguo. Establecida la cercanía, se ajusta un plano de regresión al agrupamiento, y así se le asigna la normal del plano a dicho punto. De esta manera se corrige las normales de los puntos ambiguos que se encuentran en las discontinuidades.

\section{RESULTADOS Y DISCUSIÓN}

Para mostrar el comportamiento del procedimiento de eliminación de valores atípicos, se tomó un objeto que contiene regiones con características geométricas variables. El objeto de la Figura 3 fue alterado con ruido Gaussiano del $10 \%$ para simular los valores atípicos. La figura $3 a$ muestra el objeto con la presencia de datos atípicos. El resultado de la aplicación del método de eliminación de ruido se muestra en la Figura 3b. 


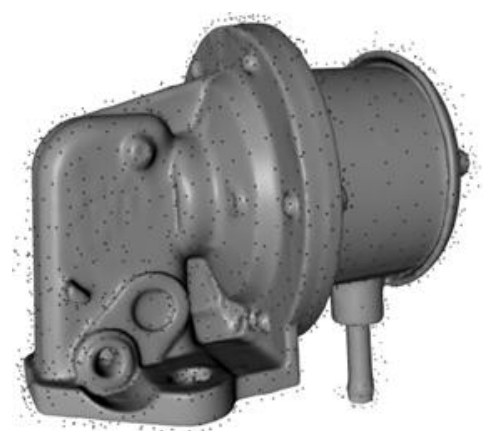

a)

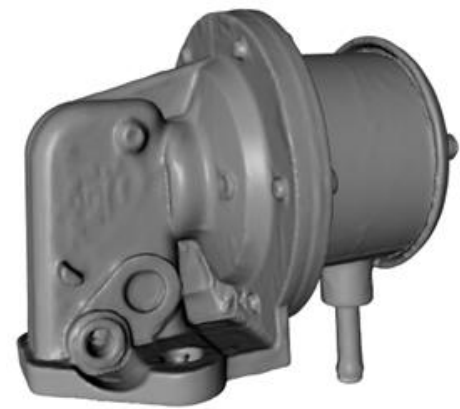

b)

Fig. 3. Aplicación del método de eliminación de valores atípicos, a) objeto con presencia de valores atípicos y b) resultado de la aplicación del método.

En relación con la estimación de las normales se consideró un conjunto de puntos que fueron muestreados de las curvas $r=k \frac{x^{3}}{6}$, en el intervalo $x \in[-1,1]$, para diferentes valores de la curvatura. Como la normal exacta a la curva está orientada en dirección del eje $y$ su valor está dentro del intervalo[0,1]. Se puede estimar el error entre la normal verdadera y la estimada por medio del ángulo formado por éstas, para ello se le ha adicionado una cantidad de ruido a las coordenadas $y$.

En la figura 4a, se muestra el error como una función del tamaño del vecindario $r$ (con $r=\max _{i}\left\|\left(p_{i}-\bar{p}\right)\right\|$ ), cuando el ruido es del $5 \%$ para tres diferentes valores de $\mathrm{k}=0.4,0.8$ y 1.2. El gráfico muestra que a medida que el radio crece (más vecinos), el error se incrementa, esto debido a que a medida que se aumenta el tamaño del vecindario se incluyen variaciones geométricas que no son posible de modelar mediante una aproximación lineal, lo que altera la aproximación. Sin embargo, es importante anotar que en regiones con naturaleza plana, el comportamiento el contrario. Basado en esto, el interés se centró en analizar el comportamiento del error para valores muy pequeños de $r$.

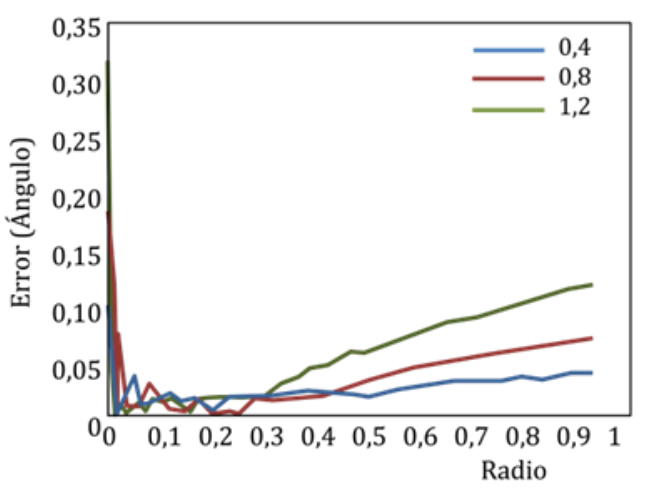

a)

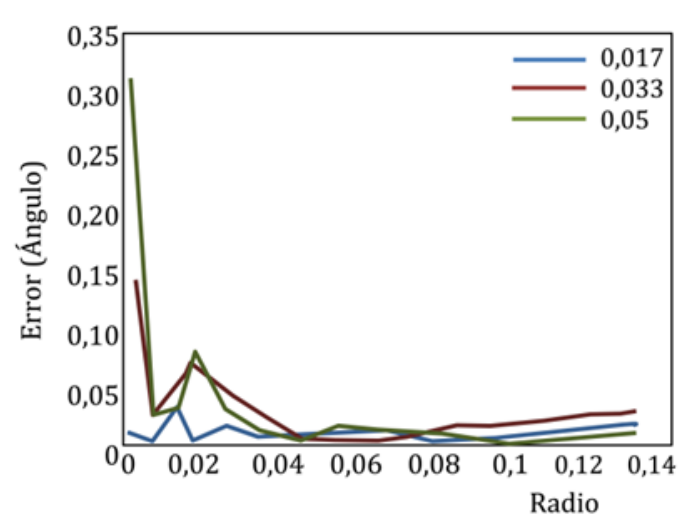

b)

Fig.4.Comportamiento del error mediante la variación del radio, a) valores de $r>0,2$ y b) valores de $r<0,08$.

La Figura 4b, muestra la estimación del error como una función del tamaño del vecindario $r$ para pequeños valores de $r$. Se observa que el error tiende a decrecer cuando $r$ incrementa. Lo que sugiere que las características geométricas de la superficie deben determinar el tamaño del vecindario, haciéndolo variable dependiendo de éstas.

Para mostrar como el método propuesto trabaja en bordes y esquinas, mostramos como fue aplicado al modelo de la Figura 5a, con ruido gaussiano $\sigma=5 \%$ del promedio de la coordenada $z$, se observa que el método maneja bien las esquinas y bordes del modelo (ver figura $5 b$ ).

La eficiencia del método también se visualiza en la aplicación de éste, en un modelo irregular como el de la figura 6a, la utilización del vecindario variable y el RPCA, hace que donde existen bordes y esquinas, los límites de la superficie quedan mejor definidos, en comparación cuando se estima la normal con vecindario fijo y PCA. En la figuras $6 \mathrm{~b}$ y $6 \mathrm{c}$, se puede observar el funcionamiento del método propuesto, tanto en modelos con alta densidad de puntos como con baja densidad. 


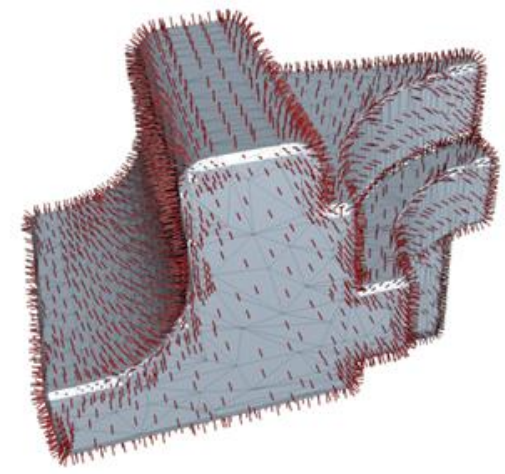

a)

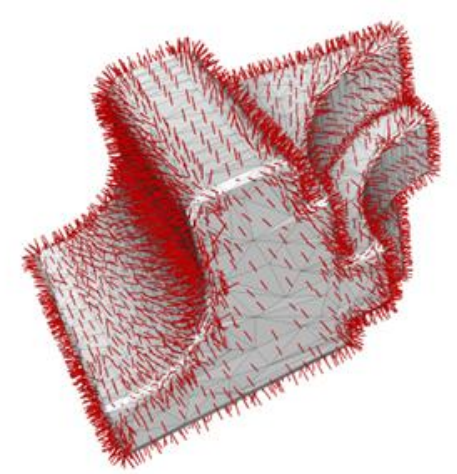

b)

Fig.5. Estimación de la normal, (a) sin ruido, (b) contaminado con ruido gaussiano.

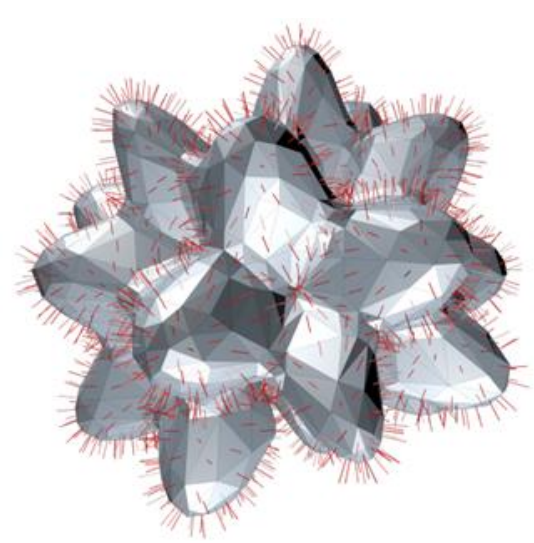

a)

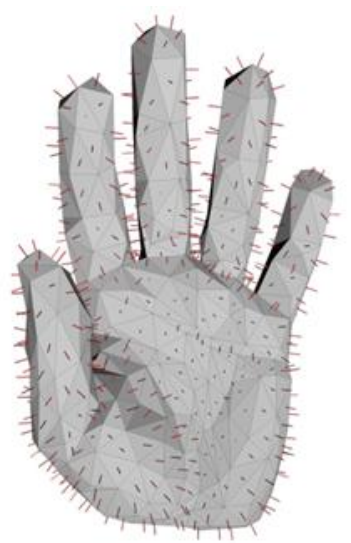

b)

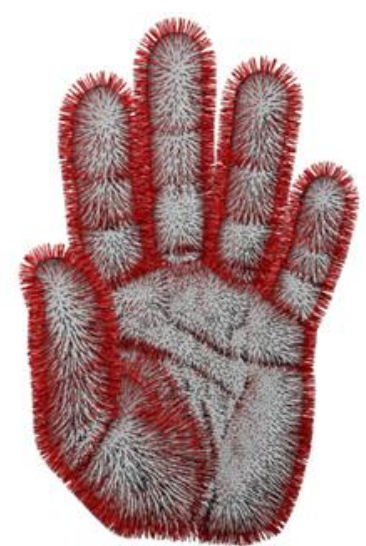

c)

Fig.6. Estimación de la normal en a) objeto irregular; (b) baja densidad de puntos; (c) alta densidad de puntos.

\section{CONCLUSIONES}

El método se apoya en el uso de una variante robusta del análisis de componentes principales RPCA, la cual por la manera de escoger los pesos, no necesita de parámetros extras ingresados por el usuario. El método propuesto utiliza un tamaño de vecindario variable alrededor de cada punto de la nube. La variabilidad depende de las características locales de la superficie que representa la nube de puntos. Se ha mostrado con resultados numéricos y visuales la validez del método propuesto mostrando como disminuye el error de la estimación cuando el tamaño del vecindario es variable a diferencia de uno fijo.

El método propuesto, tiene en cuenta los detalles finos como bordes y esquinas, de tal manera que la estimación en estas características se haga de una manera correcta. De igual forma, detecta y corrige los valores atípicos apoyados en la Distancia Robusta de Mahalanobis.

\section{REFERENCIAS}

Aleksandrov, A., Kolmogorov, A. y Laurentiev, M., La matemática: su contenido, métodos y significado, 1ra edición, Alianza Editorial, Madrid (1984).

Alexa, M., Behr, J., Cohenor, D., Fleishman, S., Levin, D. y Silva, C., Point Set Surfaces, In Proceedings of IEEE Visualization, 21-26, San Diego, California (2001).

Alliez, P., Cohen-Steiner, D., Tong, Y. y Desbrun, M., Voronoi-based variational reconstruction of unoriented point sets. In Proc. of SGP, 39-48, Aire-la-Ville, Switzerland (2007).

Bao, L., Ruwen, S., Reinhard, K., Zhiquan, C., Gang, D. y Shiyao, J., Robust normal estimation for point clouds with sharp features, Computers Graphics: 34(2), 94-106 (2010). 
Boulch, A. y Renaud, M. Fast and robust normal estimation for point clouds with sharp features, Computer \& Graphics: 31(5), 1765-1774 (2012).

Cuesta, E., Fernandez, P., Alavarez, B. y Blanco, D., Influencia del Acabado Superficial en el Digitalizado con Sensores de Triangulación por Láser. Inf. Tecnol., 19(3), 107-114 (2008).

Dey, K., Sun, J., Normal and Feature Estimation from Noisy Point Clouds, Lecture Notes in Computer Science: 4337, 21-32 (2006).

Dey, T. y Goswami, S., Provable Surface Reconstruction from Noisy Samples, Proc. 20th Annual Symposium on Computational Geometry: 35(2), 124-141 (2006).

Fleishman, S., Cohenor, D. y Silva, T., Robust Moving Least-squares Fitting with Sharp Features, TOG: 24(3), 544-552 (2005).

Filzmosera, P., Garrettb, R. y Reimann, C., Multivariate outlier detection in exploration geochemistry, Computers \& Geosciences: 31(1), 579-587 (2005).

Gumhold, S., Wang, X. y Macleod, R., Feature Extraction From Point Clouds, Proceedings 10th International Meshing Roundtable, 293-305, Sandia National Laboratories, USA (2001).

Hoppe, H., Derose, T., Duchamp, T., McDonald, J. y Stuetzle, W., Surface Reconstruction from Unorganized Points, Computer Graphics: 26(2), 71-78 (1992).

Hubert, M., Rousseuw, P. y Brannden, k., ROBPCA: A New Approach to Robust Principal Component, Analysis Technometrics: 47(1), 64-79 (2005).

Levin, D., The approximation Power of Moving Least-squares, In Math. Comp: 67(224), 1517-1531 (1998).

Mitra, J., Nguyen, A. y Guibas, L., Estimating Surface Normals in Noisy Point Cloud Data, International. Journal of Computational Geometry and Applications: 14, 261-279 (2004).

Mederos, B., Velho, L. y De Figueiredo, H., Robust Smoothing of Noisy Point Clouds. In Proc. SIAM Conference on Geometric Design and Computing, 95-106, Seattle, USA (2003).

Ouyang, D. y Feng, H., On the normal vector estimation for point cloud data from smooth surfaces, Computer-Aided Design: 37(10), 1071-1079 (2005).

Pauly, M., Gross, M. y Kobbelt, L., Efficient Simplification of Point Sampled Surfaces, In Proceedings of IEEE Visualization, 163-170, Washington, DC, USA (2002).

Risent, J., Masia, J., Lopez, J. y Ferrandiz, S. Reconstrucción Tridimensional de Superficies en el Cuerpo Humano. Inf. Tecnol. [online]. 24(5), 31-40. 2013.

Woo, H., Kang, E., Semyungkwan, W. y Lee, H. A New Segmentation Method for Point Cloud Data, International Journal of Machine Tools \& Manufacture: 24(2), 167-178 (2002). 